\title{
WS2-C03
}

\section{Reghosted 4D and 3D Reservoir Characterization Using a Broadband Monitor on a Deep Offshore Turbiditic Field}

\author{
C. Deplante* (Total E\&P Angola), F. Jeanjean (Total E\&P Angola), V. \\ Sebastiao (Total E\&P Angola) \& S. Sageder (Total)
}

\section{SUMMARY}

On a turbiditic deep offshore field covered by a recent yet conventional 4D baseline, a non-conventional route has been decided for the first monitor, to obtain seismic information related to dynamic heterogeneities but also better characterize reservoirs. The strategy decided for the first monitor (M1), one year after first oil, was to shoot broadband and process twice:

- One 4D-dedicated processing mapped to conventional (with a Fast Track and a Full Processing phase) in order to match the baseline characteristics and obtain quality 4D signal to help understanding the early dynamic behaviour of the field.

- One 3D broadband processing aiming to improve the existing seismic for reservoir characterization purposes.

The superior resolution of the 4D signal over conventional data helps pushing the interpretation below the $3 \mathrm{D}$ resolution limits and has helped identifying sedimentary features which have confirmed the interpretation model but are also difficult to map accurately. Using the 3D broadband processing results, small scale geological details previously guessed from conventional data and locally underlined by 4D signal (but only at places impacted by changes in saturation and pressure), can now be seen more clearly: a more comparable resolution is achieved for 3D and 4D. 


\section{Amsterdam '14}

\section{Introduction}

The issue of obtaining fit-for-purpose seismic data to optimize the production of a field with complex dynamic behaviour is illustrated in the case of deep offshore turbiditic reservoirs, consisting of a series of upper Miocene channel complexes. On this field covered by a recent yet conventional 4D baseline, a non-conventional route has been decided for the first monitor, to obtain seismic information related to dynamic heterogeneities but also better characterize reservoirs. The strategy decided for the first monitor (M1), one year after first oil, was to shoot broadband and process twice:

- One 4D-dedicated processing mapped to conventional (with a Fast Track and a Full Processing phase) in order to match the baseline characteristics and obtain quality 4D signal to help understanding the early dynamic behaviour of the field.

- One 3D broadband processing aiming to improve the existing seismic for reservoir characterization purposes.

\section{Workflow}

Despite a complicated workflow involving the generation of a reghosted dataset for 4D purposes, the final 4D data were produced in 4 months. The 3D broadband processing took 10 months overall.

\section{Results}

The 3D quality of M1 data remapped to conventional proved superior to the baseline data which suffered from more swell noise in a shallower streamer configuration. Despite baseline noise, the 4D signal is strong and exhibits a relatively low noise. The Total warping methodology enabled to deliver a very high resolution relative velocity change $(\mathrm{dV} / \mathrm{V})$ volume. The current workflow of $4 \mathrm{D}$ interpretation provided key information to understand the global pattern of pressure and fluid changes under current production. It has however highlighted local complexity and the importance of subseismic drains and barriers. The high $\mathrm{S} / \mathrm{N}$ ratio of $4 \mathrm{D}$ signal enables to reach a $4 \mathrm{D}$ resolution which in theory and in practice, exceeds the one of the $3 \mathrm{D}$, as illustrated below.

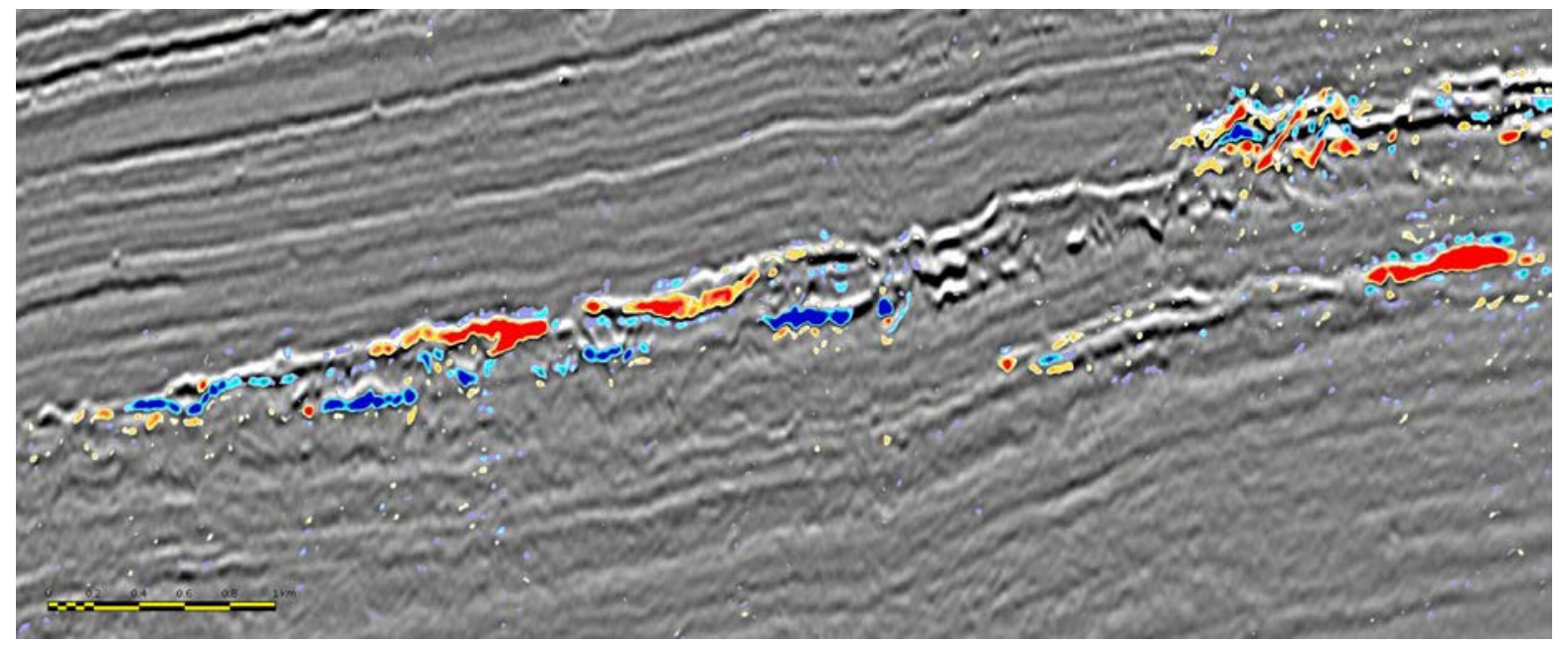

Figure 1 Example of $4 D$ relative velocity change $(d V / V)$ obtained by warping the reghosted $M 1$ data and the conventional baseline (overlain) on Miocene reservoir. The water rise appears as a blue, positive $d V / V$, whereas onsets of overpressure in water and depletion-induced gas exsolution in oil are characterized by negative $d V / V$, in red. The $3 D$ seismic resolution remains inferior to the $4 D$ one.

The same section is then shown with 4D signal overlain on broadband data with richer low and high frequencies. The broadband dataset (reflectivity and pseudo-impedance) better captures the subtle sedimentary features, which 4D proves to be acting as drains, interpreted as elementary channels. Both interpretation elements now reinforce each other and some ambiguities about what appears as possible 4D noise can now be more clearly associated with fine sedimentological or structural detail. 


\section{Amsterdam '14}

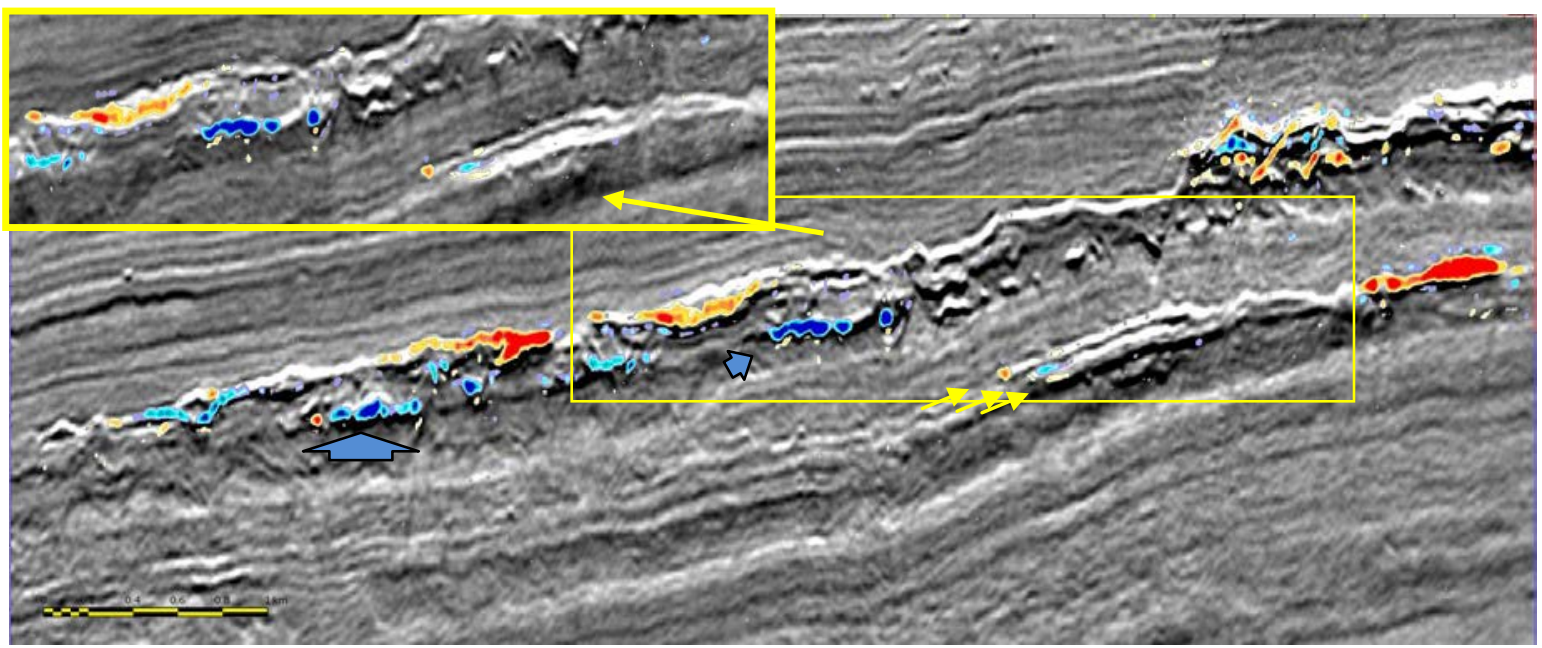

Figure 2 Same section, with broadband $4 D$ signal. The Pseudo-impedance caption confirms that the $3 D$ resolution achieved now matches the most subtle $4 D$ features, providing greater detail for WOC rise or other communication at the base of the upper reservoir and revealing three individual thin reservoirs with individual signs of overpressure or water injection in the lower reservoir.

The 4D signal confirms that an improved delineation of faulting, reservoir facies and fluids can now be achieved using the broadband data, which becomes the new reference for detailed 3D reservoir interpretation. Subsequent inversion and reservoir characterization work is ongoing.

\section{Comments}

The superior resolution of the 4D signal over conventional data helps pushing the interpretation below the 3D resolution limits and has helped identifying sedimentary features which have confirmed the interpretation model but are also difficult to map accurately.

The 3D broadband data provides a sharper image. Small scale geological details previously guessed from conventional data and locally underlined by 4D signal (but only at places impacted by changes in saturation and pressure), can now be seen more clearly on 3D. With the broadband data, a more comparable resolution is achieved for $3 \mathrm{D}$ and 4D. A more deterministic detailed reservoir interpretation, characterization and filling of geomodel properties now seems at hand.

This case study demonstrates that today's broadband acquisition and processing technology enables to confidently remap broadband data to conventional in a timely manner for conventional 4D applications, while retaining the intrinsic broadband benefits. Overall, this first monitor was an operational and technical challenge, turned into a success story of one survey to fulfill two equally important objectives addressing the two venues of field geophysics: reservoir characterization and 4D.

\section{Acknowledgements}

The authors would like to thank Sonangol Concessionaire, Total E\&P Angola, and its Block-17 associated partners, Statoil, Esso and BP for the authorization to publish this work.

\section{References}

Gomes, C., Bovet, L., Deplante, C., Tchikanha,. S. and Zamboni, E., 2013. Broadband experience on deep offshore oil field development: Angolan example, B17/CLOV project, SEG Expanded Abstract 